\section{Les protéines $\mathrm{CCN}$ : quand multimodulaire rime avec multifonctionnel}

\section{Bernard Perbal}

> La famille CCN comporte des protéines qui sont impliquées dans la régulation de phénomènes biologiques fondamentaux lors de la multiplication cellulaire normale et pathologique. L'implication de ces protéines dans la signalisation cellulaire associée à l'adhérence, la migration et la prolifération en font des éléments clés de la régulation de la croissance normale. Les contrôles négatif et positif que ces protéines effectuent vis-à-vis de la prolifération et de la différenciation cellulaire dans les conditions normales sont perturbés dans de nombreuses maladies et en particulier dans plusieurs exemples de tumeurs cancéreuses. Nous analyserons dans cet article les bases structurales qui ont conduit au regroupement de ces protéines en une même famille et qui sous-tendent leurs activités biologiques multiples. <

> Le contrôle de la croissance cellulaire normale requiert l'action concertée de nombreuses voies de signalisation faisant intervenir des cascades réactionnelles qui impliquent différents types de protéines dont la variété de mode d'action a été révélée au cours des deux dernières décennies.

L'existence d'un nouveau groupe de régulateurs de la croissance et de la différenciation cellulaires, a été mise à jour par la découverte (séquentielle) de gènes constituant ce qu'il est maintenant convenu de dénommer «la famille CCN ». Cet acronyme a été originellement proposé [1] à la suite d'une analyse informatique des séquences nucléotidiques des gènes cyr61 (cystein rich 61), ceflo (chicken embryo fibroblast10) [2, 3], fispl2 (fibroblast inducible secreted protein 12), ctfg (connective tissue growth factor) [4-6] et nov (nephroblastoma overexpressed) $[7,8]$. Cette analyse confirmait la très grande parenté structurale de ces gènes et, d'autre part, jetait les bases de la reconnaissance de quatre modules structuraux communs aux protéines pour lesquelles ils codent [5]. La découverte plus récente des

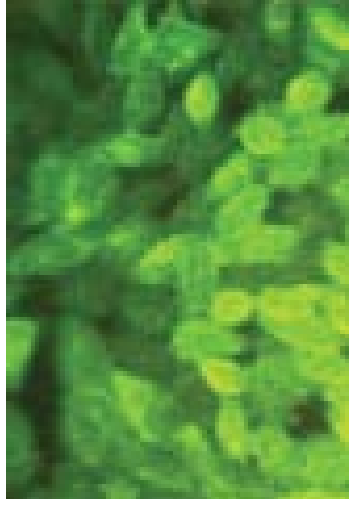

UFR de Biochimie, Université Paris 7 - D. Diderot, 2, place Jussieu, 75005 Paris, France. perbal@ccr.jussieu.fr

gènes $\varepsilon l m 1, r C O P l$ et de trois gènes WISP (wint l- induced secreted protein) [9-11] partageant le même type d'organisation multimodulaire a consolidé la notion de nouvelle famille de régulateurs, et a étendu la diversité des fonctions biologiques attribuées aux protéines CCN.

\section{La famille CCN}

Chez l'homme, on connaît actuellement six gènes (et protéines) CCN. Nous suivrons dans cet article, les recommandations de nomenclature (Tableau I) proposées par le Comité Directeur de I'International CCN Society (http://www.ccnsociety.jussieu.fr) [12].

Le gène ccnl est un gène « immédiat-précoce » dont l'expression est stimulée par les facteurs de croissance [2], le TGF $\beta$ [4], ou la transformation oncogénique par le virus du sarcome de Rous (RSV) [5]. Le gène ccn2 est aussi induit par le TGF $\beta$. La protéine pour laquelle il code possède une activité mitogénique et chimiotactique [6]. Le gène ccn3 [7] est un des sites d'intégration du virus auxiliaire de la myéloblastose aviaire (MAV) qui induit chez la poule des néphroblastomes dont les caractéristiques anatomopathologiques les apparentent aux tumeurs de Wilms [8]. La situation rencontrée 


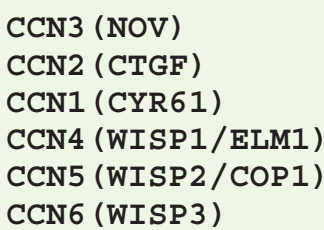

1

1

1

1

1

1 m
mqsvqstsfclrk taasmgpv ssria mrwflpwtlaavt rgtp giglif stlliag

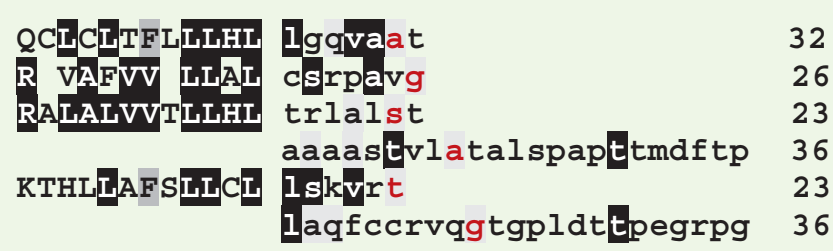

CONSENSUS

CCN3 (NOV) 33

CCN2 (CTGF) 27

CCN1 (CYR61) 24

CCN4 (WISP1/ELM1) 37

CCN5 (WISP2/COP1) 24

CCN6 (WISP 3 )
37 evsdapqrk
QFCP PC RCP QPPPRCPPGVSLVLDGCGCCKVCA

QRCP PQC PGRCP ATPPTCAPGVRAVLDGCSCCLVCA 68

QNCS GPC RCPAEPAPRCPAGVSLVLDGCGCCRVCA 61

CP AAC HCP LEAPKCAPGVGIVRDGCGCCKVCA 57

QFCK WPC ECP PSPPRCPLGVSIITDECECCKMCA 80

QLCP tPC TCP WPPPRCPLGVPLVLDGCGCCRVCA 57

QFCH WPC KCP QQKPRCPPGVSLVRDGCGCCKICA 79

\section{CONSENSUS}

CCN3 (NOV)

CCN2 (CTGF)

CCN1 (CYR61)

CCN4 (WISP1/ELM1)

CCN5 (WISP2/COP1)

CCN6 (WISP3)
KQLGESCSEADPCDPH KGLYCDYGADRP RY TGV CTA VE GVPC

69 RORGESCSDLEPCDES SGLYCDRSAD PsNO TGI CTA VE GDNC 62 KQLGELCTERDPCDPH KGLFCDFGSPAN RK IGV CTA KD EAPC $58 \mathrm{KQLNEDCSKTQPCD} \mathrm{HtKGLECNFGASST} \mathrm{AL} \mathrm{KGI} \mathrm{CRA} \mathrm{q} \mathrm{SE} \mathrm{GRPC}$ 81 QQLEDNCTEAAICDPH RGLYCDYSGDRP RYaIGV C A q VV GVGC 58 RRLGEPCDQLHVCDAS QGLVCQPGA GP GG RGALCL 1 AEdDSSC 80 KOPGEICNEADLCDPH KGLYCDYSVDRP RYeTGV C A YlV AVGC
110 103 100 123 100 122

\section{CONSENSUS}

CCN3 (NOV)

CCN2 (CTGF)

CCN1 (CYR61)

CCN4 (WISP1/ELM1) 124 CCN5 (WISP2/COP1) 101 CCN6 (WISP3)

\section{3}

EFNGVIYRNGESFQPNCKYQCTCIDGAIGCVPLC DVRLPNPDCPHP

111 VFDGVIYRSGEKFOPSCKFQCTCRDGQIGCVPRC q1 DVLLPEPNCPAP 104 IFGGTVYRSGESFOSSCKYOCTCLDGAVGCMPLC sm DVRLPSPDCPFP 101 EYNSRIYQNGESFQPNCKHQCTCIDGAVGCIPLC pq ELSLPNLGCPNP VLDGVRYNNGQSFOPNCKYNCTCIDGAVGCTPLC I RVRPPRLWCPHP VNGRLYREGETFQPHCSIRCRCEDGGFTCVPLC se DVRLPSWDCPHP EFNQVHYHNGQVFOPNPLFSCLCVSGAIGCTPLF ip kLagsh C
158 151 148 170 148 158

\section{CONSENSUS}

CCN3 (NOV)

CCN2 (CTGF)

CCN1 (CYR61)

CCN5 (WISP2 / COP1) 149

CCN6 (WISP3)

152 149
RRVEVPGKCCEEWVC DED

159 RKVEVPGECCEKWICgPDEE dslggltlaayrpeatlgvevsdssv RVKLPGKCCEEWVC RLVKVTGQCCEEWVC RRVSIPGHCCEQWVC RRVEVLGKCCPEWVC sgakgGKksdQsnC

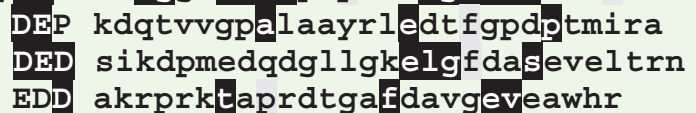

EDD akrprktaprdtgafdavgeveawhr GQG gglgtqplpaqgpqfsglvsslppgv slepllqq
204

197

195

214

192

187

CONSENSUS

NCIVQTTEWSPCSKTCG

CCN3 (NOV) 205

CCN2 (CTGF) 198

CCN1 (CYR61) 196 neliavgkgsslkrlpvfgmeprilynplqgq

CCN4 (WISP1/ELM1) 215

CCN5 (WISP2 / COP1) 193

CCN6 (WISP3) 188 lstsyktmpayrnlpliwkk

NCIEQTTEWTACSKSCG 221 NCLVQTTEWSACSKTCG 214 KCIVQTTSWSQCSKTCG 244 NCIAYTSPWSPCSTSCG 231 PCPEWSTAWGPCSTTCG 209 KCLVQATKWTPCSRTCG 224 


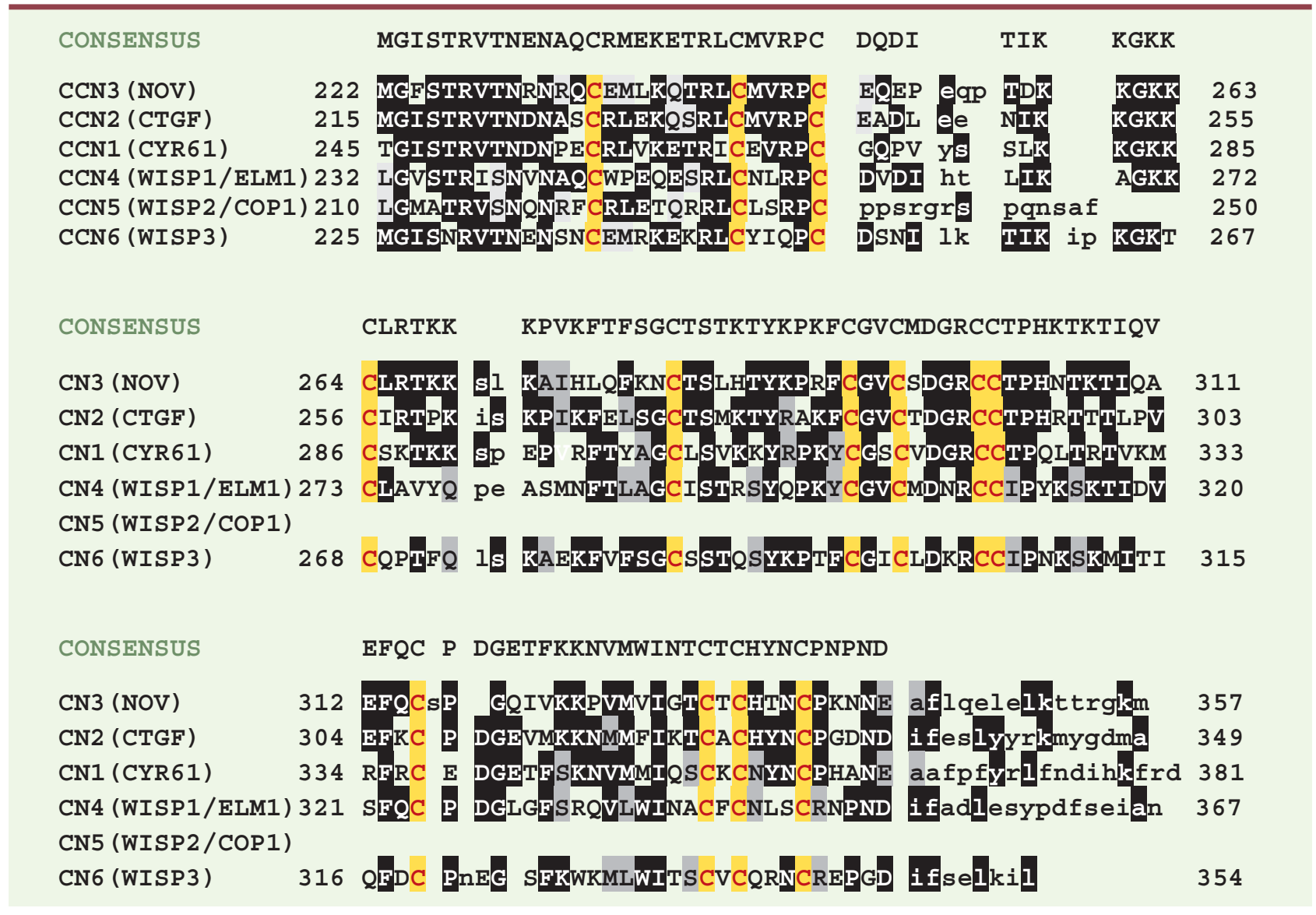

Figure 1. Conservation de la structure primaire des protéines CCN humaines. Les alignements ont été effectués avec le programme Interalign. Les résidus les plus conservés sont indiqués en blanc sur fond noir. Lorsque deux groupes de résidus différents sont conservés, le deuxième est surligné en gris. Les quatre cystéines absentes dans CCN6 sont indiquées par une astérisque. Le résidu situé en position terminale du peptide signal est indiqué en rouge pour chacune des protéines.

avec $\mathrm{CCN} 3$ constituait le premier exemple d'une protéine CCN possédant des propriétés anti-prolifératives et dont l'expression était altérée lors de la tumorigenèse.

L'analyse de gènes exprimés de manière différentielle dans des mélanomes murins à faible et haut pouvoir métastatique a permis l'isolement de ccn4, tandis que la caractérisation de gènes dont l'expression est abolie dans les fibroblastes embryonnaires murins transformés a conduit à l'identification de ccn5 [11].

Plus récemment, la caractérisation de gènes dont l'expression est stimulée dans des cellules épithéliales transformées par WNT-1 [9] a permis l'identification de wispl et wisp 2 et de ccn6 qui représentait un nouveau membre de la famille CCN.

\begin{tabular}{|l|l|}
\hline Nomenclature CCN & Autres noms utilisés dans les publications \\
\hline CCN1 & CYR 61, CEF 10, IGFBP-rP 4, bIG-MI, IGFBP10 \\
\hline CCN2 & CTGF, bIG-MI, FISP 12, IGFBP-rp 2, Hcs 24, \\
& IGFBP8, HBGF-0.8, ecogenin \\
\hline CCN3 & NOV, IGFBP-rP 3, IGFBP9 \\
\hline CCN4 & WISP-1, ELM 1 \\
\hline CCN5 & WISP-2, rCOP-1, CTGFL, CTGF-3, HICP \\
\hline CCN6 & WISP-3 \\
\hline
\end{tabular}

Tableau I. Nomenclature des protéines CCN. Des résultats récents ont indiqué que les noms attribués aux différentes protéines CCN lors de leur découverte ne sont pas toujours en adéquation avec leur fonction biologique (par exemple, CTGF n'est pas un "facteur de croissance", per se). Le comité directeur de l'International CCN Society, a proposé de dénommer ces protéines avec l'acronyme $\mathrm{CCN}$, suivi d'un nombre, attribué selon l'ordre chronologique de leur découverte. 
Homme
Souris
Rat
Caille
Poule
Xénope

CONSENSUS

Homme

Souris

Rat

Caille

Poule

xénope ex1/peptide signal

1 mqsvqstsfclrkqc LCLTFLLLH

1 mslflrkrc--.-- LCLGFLLFH

1 msvflrkqc----- LCLGFLLLH

1 mepggghslpvil-- LLLLLLLLR

1 metgggqglpv--.- I I LLL

1 mtphlalcfil-

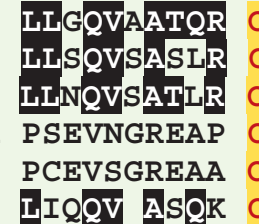

LIQQV ÂsQK ex2/domaine 1

PPCpgRCPA 45

CPSRCppKCPS 39

PSRCPSQCPS 39

CPRPCggRCPA 43

CPRPCggRCPA 41

30
CONSENSUS

Homme

Souris

Rat

Caille

Poule

xénope

CONSENSUS

Homme

Souris

Rat

Caille

Poule

xénope

CONSENSUS

Homme

Souris

Rat

Caille

Poule

xénope

EPPTCAPGVRAVLDGCSCCLVCARQRGESCSELRPCDESSGLYCDRSADP

46 TPPTCAPGVRAVLDGCSCCLVCARQRGESCSDLEPCDESSGLYCDRSADP

40 ISPTCAPGVRSVLDGCSCCPVCARQRGESCSEMRPCDQSSGLYCDRSADP

40 ISPTCAPGVRSVLDGCSCCPVCARQRGESCSEMRPCDQSSGLYCDRSADP

44 EPPRCAPGVPAVLDGCGCCLVCARQRGESCSPLLPCDESGGLYCDRGPED

42 EPPRCAPGVPAVLDGCGCCLVCARQRGESCSPLLPCDESGGLYCDRGPED

31 EPPSCAPSVLLILDGCGCCPVCAROEGESCSHLNPCQEDKGLYCEFNADP

95

89

89

93

91

80

NNQTGICMVLEGDNCVFDGVIYRNGEKFQPSCKYQCTCRDGQIGCVPRCQ - - - - - . - - - -ex3/domaine 2

96 SNQTGICTAVEGDNCVFDGVIYRSGEKFQPSCKFQCTCRDGQIGCVPRCQ 90 NNQTGICMVPEGDNCVFDGVIYRNGEKFEPNCQYFCTCRDGQIGCLPRCQ 90 NNETGICMVPEGDNCVFDGVIYRNGEKFEPNCQYHCTCRDGQIGCVPRCQ 94 GGGTGICMVLEGDNCVFDGMIYRNGETFOPSCKYQCTCRDGQIGCLPRCN 92 GGGAGICMVLEGDNCVFDGMIYRNGETFQP SCKYQCTCRDGQIGCLPRCN 81 RMETGTCMALEGNSCVFDGVVYRNRESFOPSCKYHCTCLNGHIGCVPRCN

Homme

Souris

Rat

Caille

Poule

Xénope

\section{LDLLLPGPDCPAPRKVEVPGECCEKWVCGPDEE}

146

140

140

144

142

131

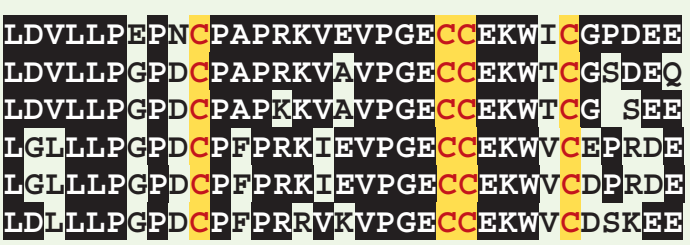

PEATLGVEVSDSSINCIEQTTEWSACSKSCGMGF STRVTNRNQQCEMVKQ - - ex4/domaine 3

191

PEATLGVEVSDSSVNN

PEATVGVEVSDSSINCIEOTTEWSACSKSCGMGVSTRVTNRNRO CFMVKO PEATVGVELSDSSINCIEQTTEWSACSKSCGMGLSTRVTNRNLQCEMVKQ QEATLGIDVSDSSANCIEQTTEWSACSRSCGMGF STRVTNRNQQCEMVKQ QEATLGIDVSDSSANCIEQTTEWSACSKSCGMGF STRVTNRNQQCEMVKO PEATLGIDASDTSFACIAQTTEWSACSKTCGMGVSS
TLGGLALAAYR

190

187

184

188

186

175
240

237

234

238

236

225 
CONSENSUS

Homme

Souris

Rat

Caille

Poule

Xénope

\section{KPRFCGVCSDGRCCTPHNTKTIQVEFQCPQGQIVKKPVMVIGTCTCHGNC}

288 KPRFCGVCSDGRCCTPHNTKTIQAEFQCSPGQIVKKPVMVIGTCTCHTNC

285 KPRFCGVCSDGRCCTPHNTKTIQVEFQCLPGEIIKKPVMVIGTCTCYSNC

282 KPRFCGICSDGRCCTPFNTKTIOVEFQCLPGQIIKKPVMVIGTCTCHSNC

284 KPRYCGLCNDGRCCTPHNTKTIOVEFRCPQGKFLKK PMMIINTCVCHGNC

282 KPRYCGLCNDGRCCTPHNTKTIQVEFRCPQGKFLKKPMMIINTCVCHGNC

273 KPKFCGQCSDGRCCTPHSTKTMHVEFVCPQKRIVKKPVMVISTCVCHYNC
337

334

331

333

331

322

CONSENSUS

PQNN EAFFQELELKTSRGKI

Homme

338

Souris

Rat

335

Caille

334

Poule

332

Xénope

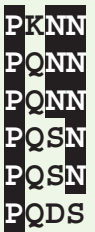

EAFLQELELKTTRGKM
EAFLQDLELKTSRGEI
EAFLQELELKT SRGEM
NAFFQPLDPMS SEAKI
NAFFQPLDPMSSEAKI

357

354

351

353

351

343

Figure 2. Conservation de la structure primaire des protéines CCN3(NOV) au cours de l'évolution. Seuls les résidus présents chez l'homme et conservés dans les autres espèces sont surlignés. Noter que dans plusieurs cas, certains résidus peuvent être conservés chez les oiseaux et les rongeurs, mais ne pas être présents chez l'homme. La très grande conservation inter-espèces suggère une fonction commune. Les jonctions des exons codant les différents domaines constitutifs de la protéine CCN3 humaine sont indiquées par les astérisques situés au-dessus de la séquence humaine .

\section{Parenté structurale des protéines de la famille CCN}

L'alignement des séquences primaires (Figure 1) montre la très grande identité structurale des différentes protéines CCN. On remarque en particulier la conservation de 38 cystéines dont la distribution (12, 10, 6 et 10 résidus) au sein des quatre modules constituant les protéines $\mathrm{CCN}$ suggère qu'elles sont engagées dans des ponts disulfures attribuant aux protéines CCN une structure compacte. La conservation de plusieurs résidus proline suggère qu'ils jouent aussi un rôle important dans le maintien d'une structure secondaire compacte.

Nous avons montré que les 38 cystéines qui sont maintenues dans la protéine CCN3 après sa maturation, le sont à une position qui est conservée, à la fois au sein des différentes protéines CCN humaines (Figure 1), et dans les différentes espèces (du xénope à l'homme) au cours de l'évolution (Figure 2). Une situation identique a été décrite dans le cas des protéines CCN1 et CCN2 [13-15]. La présence de blocs de résidus communs à toutes les protéines $\mathrm{CCN}$ suggère des parentés fonctionnelles. Cependant, ces analyses font aussi ressortir des caractéristiques structurales propres à chaque protéine $\mathrm{CCN}$, qui sous-tendent probablement leurs propriétés biologiques différentes (voir plus loin).

\section{Structure multimodulaire des protéines CCN}

Les protéines CCN contiennent un peptide signal et sont formées de quatre modules structuraux présentant une identité partielle avec : (1) les IGFBP (IGF binding proteins : protéines se liant aux IGF [insulin-like growth factors]) ; (2) le facteur von Willebrand; (3) la thrombospondine de type 1 ; et (4) des facteurs de croissance contenant un motif cystine knot . Cette organisation structurale constitue le critère d'appartenance à la famille CCN (Figure 2).

\section{Peptide signal}

La présence d'un peptide signal à l'extrémité amino-terminale des protéines CCN avait suggéré qu'elles étaient sécrétées. Effectivement, la protéine $\mathrm{CCN} l$ produite par des fibroblastes de souris (BALBC/3T3) en culture est sécrétée mais est associée, de manière très stable (avec une demi-vie supérieure à 24 heures), à la matrice extracellulaire au sein de laquelle elle pourrait être sequestrée [16] et, de manière transitoire, à la surface cellulaire [17, 18]. Elle est aussi détectée dans la fraction intracellulaire mais n'est pas détectée dans le milieu de culture.

La protéine CCN2 est présente dans le milieu conditionné des cellules de la veine du cordon ombilical (HUVEC) et subit une maturation dans le réticulum endoplasmique, ce qui est en accord avec la présence d'un peptide signal [5, 6]. La protéine CCN2 produite dans des conditions normales, par les fibroblastes de prépuce humain et par les cellules de tissus conjonctifs murins, est associée à la surface cellulaire. Elle est sécrétée très inefficacement et est très stable, alors que la protéine homologue murine FISP12 qui est très efficacement sécrétée par les fibroblastes de la lignée NIH3T3, est très instable dans le milieu. 


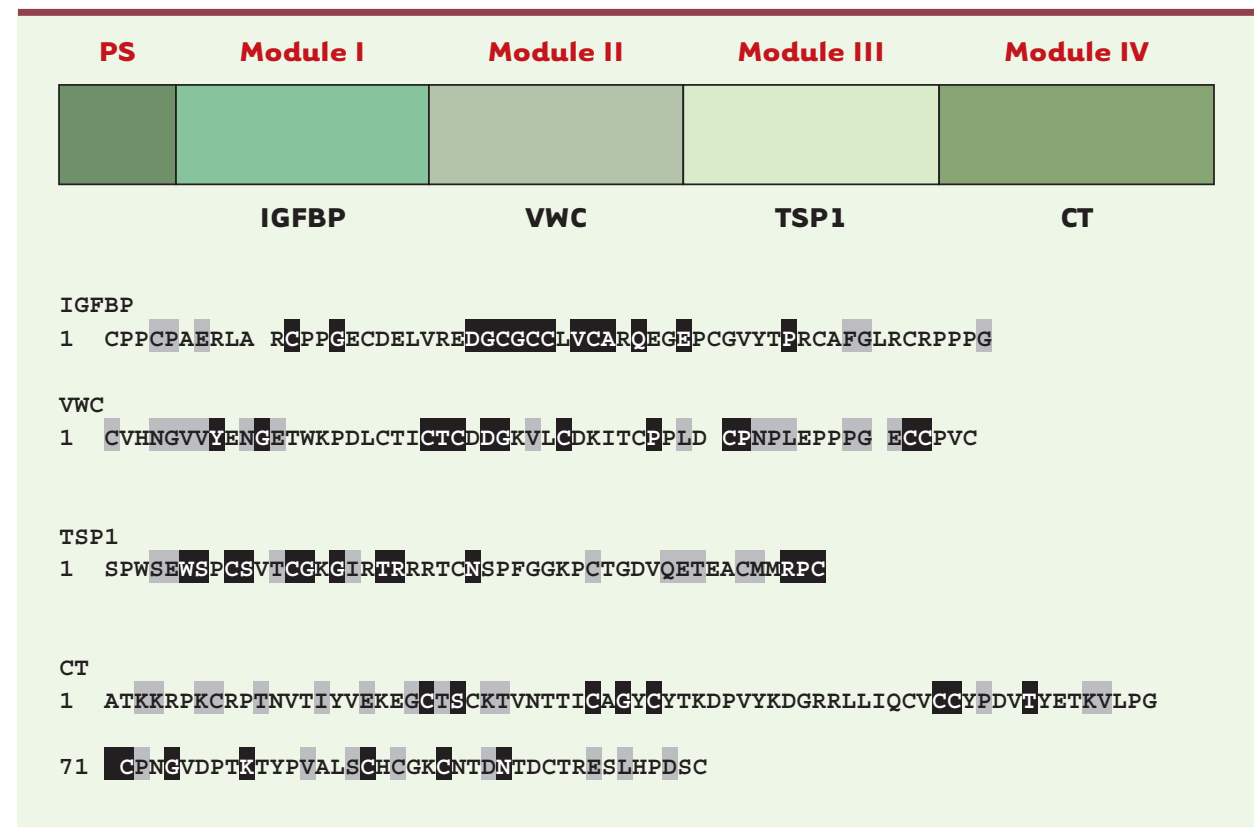

Figure 3. Organisation mosaïque des protéines $C C N$. Le schéma représente l'organisation des quatre domaines constituant les protéines $\mathrm{CCN}$. Les séquences consensus des modules sont indiquées pour faire ressortir la conservation de certains résidus (surlignés en noir) au sein des différentes protéines CCN. Les résidus surlignés en gris sont conservés dans quelques protéines seulement. PS : peptide signal.

La protéine CCN3 est associée à la matrice extracellulaire [19]. Elle est sécrétée efficacement dans le milieu de culture des fibroblastes embryonnaires de poule, de diverses cellules épithéliales humaines, et de glioblastomes humains où elle est très stable (avec une demi-vie supérieure à 24 heures). Elle est également retrouvée de manière transitoire à la surface des cellules de corticosurrénalome ( $\mathrm{NCl}-\mathrm{H} 295 \mathrm{R})$ [20] ainsi que dans les fractions cytoplasmiques et nucléaires de cellules HeLa (carcinome du col de l'utérus) et 143 (ostéosarcome humain) [21]. La protéine CCN4 murine est sécrétée très efficacement par les cellules de mélanome K-1735 [11].

Ces différentes situations reflètent sans doute une association plus ou moins forte des protéines CCN à un (ou des) récepteur(s) cellulaire(s). Les quantités de protéines CCN présentes dans les milieux de cultures pourraient varier avec l'état physiologique des cellules ou être modulées par les interactions des protéines $\mathrm{CCN}$ avec d'autres protéines membranaires et de la matrice extracellulaire.

\section{Les modules constitutifs des protéines CCN \\ Module I}

Ce module montre une identité partielle avec les IGFBP. Sur la base de la comparaison de la séquence des protéines CCN1 de souris, CCN2 humaine et $\mathrm{CCN} 3$ de poule, il avait été proposé que les protéines CCN se lient aux IGF. Par le jeu des espacements variables d'acides aminés effectués par les programmes de comparaison de séquences, il est possible d'aligner 11 cystéines des IGFBP avec 11 cystéines des protéines CCN [22]. Cependant, un examen attentif des séquences révèle que cet alignement ne correspond pas à une réalité structurale car il repose sur un décalage des séquences de CCN1 et de CCN2 qui ne respecte pas la conservation des résidus au sein des protéines CCN (Figure 4). La différence d'organisation des extrémités amino-terminales des protéines $\mathrm{CCN}$ est également révélée par les prévisions de structure secondaire, effectuées selon la méthode de Chou et Fasman [23], et qui attribuent aux protéines $\mathrm{CCN} 3,4-6$ une structure terminale riche en feuillet $\beta$, absente dans les autres membres de la famille CCN.

Des expériences de pontage covalent ont suggéré que les protéines $\mathrm{CCN} 2$ et $\mathrm{CCN} 3$ entières puissent se lier aux IGF $[24,25]$. Ces résultats sont en contradiction avec ceux que nous avions obtenus lors d'expériences de ligand blot et qui n'avaient pas permis de détecter d'interaction entre les IGF et CCN3 [26]. La très faible affinité des IGF vis-àvis de la protéine CCN3 intacte reste controversée et doit être confirmée. Nous avons proposé que les isoformes tronquées décrites par le groupe de Brigstock pour CCN2 [13] et par notre groupe pour CCN3 [21, 27] se lient efficacement aux IGF et que la protéolyse de ces protéines joue un rôle important dans la modulation de leur activité biologique. II a maintenant été établi que l'extrémité amino-terminale de CCN3 est capable de se lier à l'insuline avec une affinité beaucoup plus grande que celle des protéines IGFBP1-6 [22]. La protéolyse de CCN3 démasquerait des activités de liaison vis-à-vis de ligands pour lesquels ces protéines manifestent une faible affinité dans leur conformation native.

\section{Module II}

II présente une similitude importante avec le motif répété $C$ de la protéine de von Willebrand (Figure 3). Quatre cystéines sont absentes dans le module II de CCN6. II est pro- 
bable que ces cystéines participent à la formation de ponts disulfures propres à cette région et que leur absence ait des conséquences importantes pour les propriétés biologiques des protéines CCN. Ce motif est également retrouvé dans plusieurs autres protéines telles que le collagène $\alpha$, les thrombospondines 1 et 2, la protéine SOG (short gastrulation), de drosophile et des mucines.

Le module II pourrait être impliqué dans des processus d'oligomérisation protéique précédés par une étape de dimérisation. Les résultats obtenus dans notre équipe avec la protéine CCN3 et par différents groupes pour les autres membres de la famille CCN [28] montrent que les protéines $\mathrm{CCN}$ forment des oligomères de haut poids moléculaire. L'implication du module IV dans la dimérisation homo- et hétérotypique des protéines CCN (voir plus loin) suggère que les modules II et IV coopèrent dans l'assemblage multimérique de ces protéines. II est possible que la capacité d'oligomérisation de CCN6 soit affectée par l'absence des quatre cystéines du module II et que l'absence du module IV dans CCN5 compromette ses capacités de dimérisation et d'oligomérisation. La seconde partie du module II pourrait jouer le rôle de charnière entre les deux groupes de deux modules (I-II et III-IV) car elle ne contient aucune cystéine [14].

\section{Module III}

II présente une grande similitude avec le motif WSXCSXXCG de la thrombospondine de type 1 qui jouerait un rôle important dans l'attachement cellulaire. Le module III présente également une similitude élevée (5 cystéines alignées sur les 6 du module) avec d'autres protéines (properdine, Fspondine, protéine circumsporozoïte) impliquées dans l'interaction des cellules avec la matrice extracellulaire et contenant une ou plusieurs répétitions de ce type. La présence de ce module dans les protéines CCN a fait penser qu'elles interagissent avec des constituants de la matrice extracellulaire et qu'elles interviennent dans les processus régulateurs de la prolifération.

\section{Module IV}

La conservation des résidus au sein de ce module est moins stricte que pour les autres modules. Six des cystéines présentes dans ce module sont susceptibles d'adopter une structure dite en «nœud à cystines » (cystin knot) qui permet la formation d'homo- et d'hétérodimères dans le cas de plusieurs facteurs de croissances tels que le NGF (nerve growth factor), le TGF $\beta$ (transforming growth factor $\beta$ ) et le PDGF (platelet derived growth factor). Nous avons établi que ce module est suffisant pour permettre l'interaction de CCN3 avec la fibuline $1 \mathrm{C}$ ainsi que les protéines CCN3 et CCN2 [27].

\section{Fonctions des protéines CCN}

Dans des conditions normales, les protéines CCN participent à la régulation de processus biologiques. Elles interviennent aussi dans les conditions traumatiques, lors de la cicatrisation et dans les pathologies fibrotiques. Enfin, un

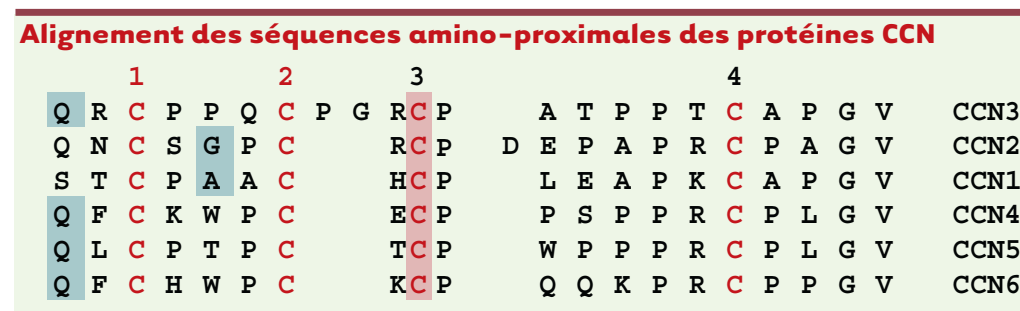

Décalage imposé pour aligner les séquences CCN et IGFBP

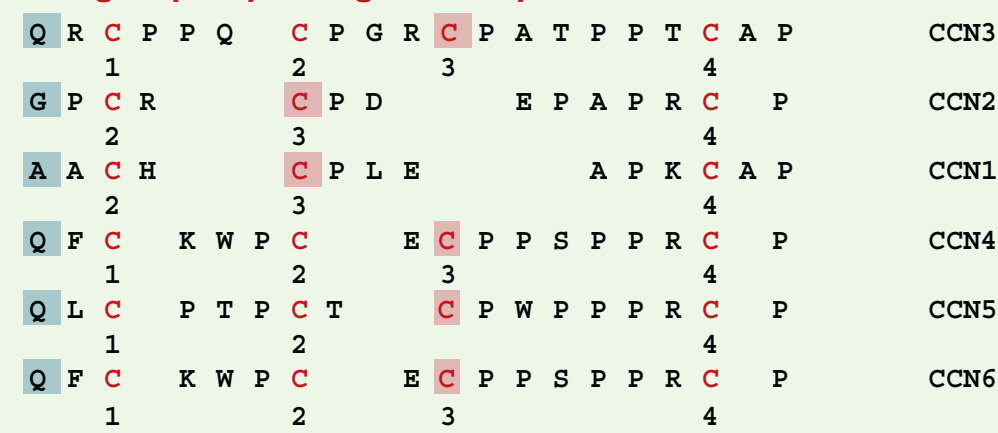

Alignement des séquences amino-proximales des IGFBP

\begin{tabular}{|c|c|c|c|c|c|c|c|c|c|c|c|c|c|c|}
\hline C & $\mathbf{P}$ & $\mathbf{P}$ & & C & $\mathbf{P}$ & A & $\mathbf{E}$ & $\mathbf{R}$ & L & A & $\mathbf{R}$ & C & $\mathbf{P}$ & CONSENSUS IGFBP \\
\hline C & $\mathbf{P}$ & $\mathbf{P}$ & & $\mathrm{C}$ & $\mathbf{T}$ & P & $\mathbf{E}$ & $\mathbf{R}$ & L & A & A & $\mathrm{C}$ & $P$ & IGFBP 2 \\
\hline C & $\mathbf{E}$ & P & & C & $\mathrm{D}$ & A & $\mathbf{R}$ & A & L & A & $Q$ & $\mathrm{C}$ & P & IGFBP 3 \\
\hline C & & $\mathbf{P}$ & G & $\mathrm{C}$ & G & Q & G & $\mathrm{V}$ & Q & A & G & C & $\mathbf{P}$ & IGFBP 6 \\
\hline 1 & & & & 2 & & & & & & & & 3 & & \\
\hline
\end{tabular}

Figure 4. Alignement des extrémités amino-proximales des protéines $\mathrm{CCN}$ et des IGFBP.

L'alignement de cystéines des protéines CCN permet aussi de faire ressortir l'alignement des autres résidus (haut de la figure). L'alignement proposé pour faire coïncider les cystéines des protéines CCN et des IGFBP (deuxième partie de la figure) repose sur un décalage des extrémités amino-terminales des protéines CCN2 et $\mathrm{CCN} 1$ (visualisé par les cystéines surlignées en jaune et les résidus surlignés en bleu) qui ne respecte pas la conservation structurale des protéines CCN représentée en haut de la figure. La différence structurale des extrémités amino-terminales des protéines CCN peut jouer un rôle important vis-à-vis des fonctions biologiques qu'elles sont susceptibles de partager avec les IGFBP. 
nombre croissant d'observations expérimentales attribue aux protéines $\mathrm{CCN}$ un rôle important dans la tumorigenèse. La détection des protéines $\mathrm{CCN} 1, \mathrm{CCN} 2$ et $\mathrm{CCN} 3$ dans le système vasculaire au cours du développement a suggéré que ces protéines interviennent dans la régulation de l'angiogenèse $[12,14,28,29]$. Cela a été confirmé par la capacité des deux premières d'induire la néovascularisation des cornées de rat [30] et l'interaction de ces protéines avec l'intégrine $\alpha v \beta 14$. L'effet angiogénique des facteurs de croissance tels que le bFGF et le TGF $\beta$ met en jeu la production des protéines CCN [14]. L'invalidation du gène ccnl chez la souris conduit à des altérations de la vascularisation des membranes extra-embryonnaires et du placenta, qui aboutissent à une importante mortalité prénatale [28].

Les protéines CCN interviennent aussi dans les processus d'ossification et de chondrogenèse. Lorsqu'elles ont été recherchées, les protéines $C C N$ ont été détectées dans le cartilage hypertrophique $[12,31]$ et la protéine $\mathrm{CCN} 1$ peut induire la différenciation des cellules mésenchymateuses [32]. Les effets paracrines in vitro de la protéine recombinante CCN2 sur la prolifération et la différenciation de chondrocytes, d'ostéoblastes et de cellules endothéliales suggèrent qu'elle est impliquée dans l'ossification endochondrale. La surexpression ectopique de ccn2 interfère avec la chondrogenèse articulaire et l'hypertrophie du cartilage, d'une manière qui dépend de la présence et du degré d'expression du TGF $\beta$. La protéine CCNl est détectée dans les zones de réparation de fractures osseuses, dans les chondrocytes du cartilage de conjugaison et dans les ostéoblastes en culture [28]. De la même manière, CCN2 est impliquée dans la squelettogenèse, pendant le développement prénatal, et dans la réparation des fractures osseuses [28]. L'invalidation du gène ccn2 chez la souris [28] conduit à des anomalies de développement du squelette au niveau des côtes, du sternum, des vertèbres cervicales et du crâne.

La protéine $\mathrm{CCN} 3$ est également détectée dans le squelette embryonnaire humain et dans les chondrocytes au cours du développement normal chez l'homme et chez la poule [12, 28 , 33]. L'expression de nov est stimulée par PTHrP (parthyroid hormone related peptide), un régulateur de la différenciation chondrocytaire dans les plaques de croissance [28]. Il est donc probable que CCN3 joue aussi un rôle dans la biologie des chondrocytes normaux.

Des résultats récents ont attribué aux protéines $C C N$ un rôle dans la polarisation au cours du développement et dans la morphogenèse. L'expression de ccn3 est modulée par le produit du gène $W n t 1$, et la greffe de cellules $\operatorname{Cos} 7$ exprimant la protéine CCN3 dans le flanc d'embryons de poulet induit la formation de bourgeons de membres surnuméraires [28]. Ces observations confirment l'implication de CCN3 dans des interactions ectomésenchymateuses et sug- gèrent, pour la première fois, qu'une protéine de la famille CCN joue un rôle dans les processus de dorsalisation chez les oiseaux. Nous avons récemment établi qu'au cours de la formation du tube neural, l'expression du gène ccn3 est asymétrique, ce qui indique que la protéine CCN3 est également impliquée dans les processus de latéralisation [34]. Chez le triton, la protéine CCN2 joue un rôle morphogène dans les processus d'indentation interdigitale et est impliquée dans l'hypertrophie du cartilage [28]. Une surexpression de ccn2 après dénervation aboutit à une atrophie musculaire [28]. Dans ce système, CCN2 joue un rôle dans les processus inflammatoires, le remodelage de la matrice extracellulaire, la prolifération cellulaire, l'ossification endochondrale et l'apoptose associée à la morphogenèse.

\section{Protéines CCN et maladies}

L'expression des protéines CCN a été associée à des maladies non tumorales telles que les fibroses rénales et hépatiques, l'athérosclérose et les sclérodermies. Le TGF $\beta$ intervient dans le contrôle de la production de la protéine CCN2 au cours des processus pathologiques. Cependant, plusieurs résultats récents ont indiqué que cette relation n'est pas obligatoire. La protéine CCN2 complémente les activités biologiques du TGF $\beta$, mais n'est pas un médiateur de tous ses effets profibrogènes et a des effets propres qui ne sont pas liés à ceux du TGF $\beta[28,35]$.

L'identification du gène $\mathrm{CCN} 3$ avait fourni les premières indications montrant que l'altération de l'expression des protéines CCN était impliquée dans la tumorigenèse [7]. Il est maintenant établi que l'expression du gène ccnl est altérée dans les cancers mammaires où la protéine $\mathrm{CCN} 1$ jouerait un rôle important dans la progression tumorale [36]. Une altération de l'expression de ccnl a également été décrite dans le cas des cancers pancréatiques [28].

Les résultats récents obtenus par notre équipe ont mis en exergue l'implication des protéines CCN dans le développement et le maintien des tumeurs cancéreuses. Nous avons établi que l'expression de ccn3 est associée à un pronostic favorable et constitue un marqueur de différenciation tumorale utilisable en clinique pour le typage de tumeurs telles que les neuroblastomes ou les tumeurs de Wilms, alors que dans les corticosurrénalomes - dans lesquels ccn3 est exprimé de manière très variable [12] - il ne constitue pas un marqueur fiable à lui seul. Dans le cas des sarcomes d'Ewing, des carcinomes rénaux et de certaines lignées de tumeurs prostatiques, l'expression de ccn3 est associée à une capacité proliférative et à une tumorigénicité accrue [37, 38]. 


\section{Mode d'action}

L'obtention de protéines obtenues à partir de clones baculoviraux recombinants a permis d'étudier quelques effets biologiques de ces protéines. Cependant, il faut conserver à l'esprit que, dans les cellules d'insectes, ces protéines ne subissent pas toutes les modifications post-traductionnelles auxquelles elles sont soumises dans les cellules d'eucaryotes supérieurs [27]. Certaines de leurs propriétés biologiques, en particulier celles qui dépendent d'interactions avec des récepteurs ou des autres protéines solubles membranaires, peuvent nécessiter de telles modifications (exemple classique des glycosylations).

Les protéines recombinantes $\mathrm{CCN} 1$ et $\mathrm{CCN} 2$ ont toutes les deux des effets mitogéniques mais n'ont pas les mêmes modes d'action. Alors que la protéine CCN2 humaine, exerce une action directe sur la prolifération des cellules NIH3T3 [6] et des fibroblastes NRK [39], la protéine CCN1 d'origine humaine ou murine n'a pas d'activité mitogène intrinsèque; elle potentialise les effets d'autres facteurs de croissance sur les cellules endothéliales et fibroblastiques. De manière inattendue, la protéine CCN2 d'origine murine n'a pas d'effet direct sur la prolifération des cellules NIH 3T3 et des cellules HUVEC, mais agit en synergie avec les facteurs de croissance qui stimulent la synthèse d'ADN dans ces cellules [40]. Cette observation indique que les différentes activités biologiques des protéines CCN2 humaine et murine peuvent être dues à des différences structurales ou aux différentes conditions expérimentales dans lesquelles ces activités sont mesurées [14].

De manière tout à fait opposée à ces effets stimulateurs, la protéine aviaire CCN3 recombinante native induit l'arrêt de croissance des fibroblastes embryonnaires de poule lorsqu'elle est produite en grande quantité [7] et son expression maximale dans des conditions de culture normales coïncide avec l'apparition de la phase de quiescence cellulaire [41]. Cependant, les expériences d'hybridation in situ que nous avons réalisées chez la poule et chez l'homme $[33,34]$ ont établi que l'expression de CCN3 n'est pas restreinte aux cellules quiescentes et de récents résultats ont attribué à la protéine $\mathrm{CCN} 3$ un effet stimulant la croissance sur les cellules de souris NIH3T3 [42].

Les propriétés biologiques des protéines CCN semblent donc différentes selon le contexte cellulaire et la nature de facteurs avec lesquelles elles sont susceptibles d'interagir [12].

Plusieurs observations attribuent aux protéines $C C N$ un rôle dans les phénomènes d'adhérence et de migration cellulaire.

Nous avons établi que la protéine $\mathrm{CCN} 3$ interagit avec la fibuline IC [27], protéine de la matrice extracellulaire qui intervient dans le contrôle de l'adhérence.
Les protéines $\mathrm{CCN} 1$ et $\mathrm{CCN} 2$ favorisent l'adhérence de différents types cellulaires (cellules vasculaires endothéliales, fibroblastes, cellules épithéliales et plaquettes) quand elles sont pré-adsorbées sur des supports plastiques [14]. Plusieurs résultats expérimentaux indiquent que ces protéines stimulent la migration des fibroblastes NIH3T3 et des cellules endothéliales [29, 43]. Les activités chimiotactiques des protéines $\mathrm{CCN} 1$ et $\mathrm{CCN} 2$ jouent un rôle important dans l'activité angiogénique de ces protéines [14]. Des travaux récents ont montré que l'adhérence, la migration et la prolifération relayées par $\mathrm{CCN} 1$ requièrent respectivement les intégrines $\alpha 6 \beta 1, \alpha v \beta 5$ et $\alpha v \beta 3$ [44-46]. Les protéines $\mathrm{CCN} 1$ et $\mathrm{CCN} 2$ stimulent l'adhérence des plaquettes en interagissant avec l'intégrine $\alpha$ llb $\beta 3$. Ces résultats illustrent la variété d'activité des effets biologiques des protéines CCN et fournissent un support à leur spécificité cellulaire.

La similitude structurale des différentes protéines CCN suggère que d'autres membres de cette famille puissent également interagir avec les intégrines et participer aux phénomènes d'adhérence et de migration qui sous-tendent l'angiogenèse.

Nos études ont établi que la protéine CCN3 interagit avec l'intégrine $\alpha v \beta 3$ et avec l'intégrine $\alpha$ llb $\beta 3$.

Nos études ont montré que le module carboxy-terminal des protéines CCN joue un rôle important dans la régulation de l'interaction cellulaire avec les substrats. En accord avec cette hypothèse, il a été montré récemment [44] que la protéine CCNl privée de son module carboxyterminal pouvait encore stimuler les intégrines $\alpha 6 \beta 1$ et $\alpha v \beta 3$, mais ne permettait plus l'adhérence des fibroblastes humains en culture.

Il semble donc que le module carboxy-terminal, en s'associant à différents partenaires, confère aux cellules qui produisent les protéines CCN la capacité d'interagir avec les cellules voisines. Nous avons proposé [12] que la formation de tels complexes joue un rôle essentiel dans la part de la signalisation positive et négative exercée par ces protéines. La biodisponibilité des diverses protéines CCN à un site particulier constituerait un élément clé de la régulation de leur activité biologique et l'altération constitutive du module carboxy-terminal rencontrée dans la protéine CCN5 pourrait rompre cet équilibre fonctionnel.

L'élimination du module I, qui a été observée dans le cas de la mutagenèse insertionnelle de ccn3 par le virus aviaire MAV [7] a fourni le premier exemple permettant d'attribuer une base structurale à la modification fonctionnelle d'une protéine $\mathrm{CCN}$. II semble en effet que le ciblage incorrect de cette protéine tronquée interfère avec le dosage des différentes isoformes cytoplasmique et nucléaire [21, 27] de CCN3 et se traduise par l'acquisition d'un phénotype transformé. 


\section{Conclusions}

La structure multimodulaire des protéines CCN suggérait une multifonctionnalité. L'implication de ces protéines dans plusieurs processus biologiques fondamentaux au cours du développement normal et pathologique a confirmé cette prédiction.

Plusieurs situations non exclusives peuvent être envisagées pour rendre compte des multiples propriétés biologiques des protéines CCN : (1) elles sont la somme des propriétés de chacun des quatre modules; (2) elles sont propres à l'assemblage que constitue l'association des quatre modules; (3) elles sont apparentées aux protéines qui contiennent ces modules tout en possédant une spécificité qui résulte de leur structure multimodulaire. En tout état de cause, la structure mosaïque des protéines CCN représente un exemple unique de brassage d'exons ayant conduit à la réunion de quatre modules dont les propriétés biologiques potentielles peuvent fournir une base structurale à l'implication des protéines CCN dans les voies de signalisation de la prolifération et de la croissance, dans les processus biologiques liés à la coagulation sanguine et à l'angiogenèse, ainsi que dans les fonctions d'attachement et de migration faisant intervenir des processus de communication intercellulaire et avec le milieu.

Nous avons proposé un modèle [12] selon lequel la diversité des propriétés biologiques attribuées aux protéines CCN résulterait de différentes combinatoires impliquant des partenaires dont la biodisponibilité varie avec la nature et l'état de développement des tissus dans lesquelles elles exercent leurs fonctions. Selon ce modèle, les extrémités amino- et carboxy-proximales des protéines CCN seraient respectivement responsables d'activités régulatrices négatives et positives. Leur élimination, qui est rencontrée dans certaines situations biologiques, normales ou pathologiques, conférerait aux protéines correspondantes des propriétés stimulatrices ou inhibitrices de type « constitutif», pouvant résulter de leur incapacité d'interagir avec des partenaires qui seraient impliqués dans la médiation de leur activité régulatrice.

L'identification des relations pouvant exister entre la structure multimodulaire des protéines $\mathrm{CCN}$ et leurs fonctions biologiques constitue un défi scientifique remarquable car elle pourrait permettre l'utilisation de cette nouvelle famille de protéines dans les protocoles de diagnostic, de typage et de pronostic d'une médecine moléculaire en pleine expansion. $\diamond$

\section{SUMMARY}

The multimodular organization of the CNN proteins The CCN family of genes and proteins is composed of key regulators involved in the control of cell growth in normal and pathological conditions. The CCN proteins are controling cell adhesion, motility and proliferation. In pathological conditions, such as cancer development, the balance of negative and positive signals induced by these regulators is altered. In this review article we analyse the multimodular organization of the $\mathrm{CCN}$ proteins and discuss features that provide a structural basis for their multiple biological activities. $\Delta$

\section{RéFÉRENCES}

1. Bork P. The modular architecture of a new family of growth regulators related to connective tissue growth factor. FEBS Lett 1993 ; 327 : 125-30.

2. Lau LF, Nathans $D$ Expression of a set of growth-related immediate early genes in BALB :c 3T3 cells :coordinate regulation with c-fos or c-myc. Proc Natl Acad Sci USA 1987 ; $84: 1182-6$.

3. Simmons D, Levy D, Yannoni Y, Erikson RL. Identification of a phorbol esterrepressible $v$-src inducible gene. Proc Natl Acad Sci USA 1989 ; 86 : 1178-82.

4. Almendral J, Sommer D, Mac Donald-Bravo H, Burckhardt J, Perera J, Bravo R. Complexity of the early genetic response to growth factors in mouse fibroblasts. Mol Cell Biol $1998 ; 8: 2140-8$.
5. Ryseck RP, MacdonaldBravo H, Mattei MG, Bravo R. Structure, mapping, and expression of fisp-12, a growth factor-inducible gene encoding a secreted cysteine-rich protein. Cell Growth Differ 1991 ; 2 : 225-33.

6. Bradham DM, Igarashi A, Potter RL, Grotendorst GR. Connective tissue growth factor: a cysteine-rich mitogen secreted by human vascular endothelial cells is related to the SRC-induced immediate early gene product CEF-10. J Cell Biol 1991 ; 114 : 1285-94.

7. Joliot V, Martinerie $C$, rearrangements and overexpression of a new cellular gene (nov) in myeloblastosis-associated virus type 1-induced nephroblastomas. Mol Cell Dambrine G, et al. Proviral Biol 1992 ; 12 : 10-21.

\section{REMERCIEMENTS}

Les travaux effectués dans mon laboratoire ont été financés par le Comité National et les Comités du Cher et de l'Indre de la Ligue Nationale Contre le Cancer, l'Association pour la Recherche contre le Cancer, Matra Hachette et la Fondation pour la Recherche Médicale, le Ministère de l'Éducation Nationale et de la Recherche et l'Association Française contre les Myopathies. Je remercie ma femme pour son soutien constant et son aide bénévole journalière au sein du laboratoire. 


\section{NOTદ AJOUTÉદ AUX દ́PREUVES}

Depuis l'acceptation de cet article pour publication dans médecine/sciences, en septembre 2001, nous avons montré que l'expresssion de ccn3 dans les tumeurs d'Ewing constitue un facteur de risque pour le développement de métastases osseuses et pulmonaires [47]. Nous avons aussi établi que la production de la protéine CCN3 par les cellules de glioblastomes interfère avec leurs propriétés tumorigènes in vivo [48]. Ces deux exemples renforcent l'utilité de CCN3 comme outil biomédical. Plusieurs articles ont également rapporté l'altération de la progression tumorale par CCN2 dans les cancers de l'œsophage [49], son implication dans l'angiogenèse tumorale [50] et l'expression de ccnl, ccn4 et con5 dans les lignées de cancer du sein [51, 52], ainsi que dans des tumeurs primaires du sein [53]. Plus récemment, il a été démontré que WISP3 possède une activité de type suppresseur de tumeurs dans le cas des cancers inflammatoires du sein [54].

Des résultats récents ont également établi que des épissages alternatifs sont responsables de la production de protéines CCN1 - tronquée du motif thrombospondine dans des fibroblastes humains [55] stimulés par le sérum - et CCN4, tronquée du motif von Willebrand [56] dans des carcinomes gastriques. Ces observations soulignent la variété structurale des différentes protéines CCN et soulèvent des questions intéressantes en ce qui concerne le rôle biologique de ces différentes isoformes dans la biologie des cellules normales et tumorales.

8. Perbal B. Contribution of MAV-1-induced nephroblastoma to the study of genes involved in human Wilms' tumor development.

Crit Rev Oncog 1994 ; $5:$ 589-613.

9. Pennica D, Swanson TA, Welsh JW, et al. WISP genes are members of the connective tissue growth factor family that are up-regulated in Wnt-1transformed cells and aberrantly expressed in human colon tumors. Proc Natl Acad Sci USA 1998 ; $95:$ 14717-22.

10. Zhang R, Averboukh L, Zhu W, et al. Identification of rCop-1, a new member of the CCN protein family, as a negative regulator for cell transformation. Mol Cell Biol 1998 ; 18 : 6131-41.
11. Hashimoto By, ShindoOkada N, Tani M, et al. Expression of the ELMI gene, a novel gene of the CCN (connective tissue growth factor, Cyr61/Ceflo, and nephroblastoma overexpressed gene) family, suppresses in vivo tumor growth and metastasis of K- 1735 murine melanoma cells. J Exp Med 1998 ; 187 : 289-96.

12. Perbal B. Nov (nephroblastoma overexpressed) and the CCN family of genes: structural and functional issues. Mol Pathol 2001 ; $54: 57-79$.

13. Brigstock DR. The connective tissue growth factor/cysteine-rich 61/nephroblastoma overexpressed (CCN) family. Endocrinol Rev
$1999 ; 20: 189-206$

14. Lau LF, Lam SC. The CCN family of angiogenic regulators: the connection. Exp Cell Res 1999 ; 248 : 44-57.

15. Essam ED, Moussad A, Brigstock D. Connective tissue growth factor: What's in a name. Mol Genet Metab 2000 ; 71 : 276-92.

16. Kireeva M, Latinkic B, Kolesnikova T, et al. Cyr61 and Fisp12 are both ECM-associated signaling molecules: activities, metabolism, and localization during development. Exp Cell Res $1997 ; 233: 63-77$.

17. Yang GP, Lau LF. Cyr61, product of a growth factorinducible immediate early gene, is associated with the extracellular matrix and the cell surface.
Cell Growth Differ 1991; 2: 351-7.

18. 0'Brien TP, Yang GP, Sanders L, Lau LF. Expression of cyr6l, a growth factor-inducible immediate-early gene. Mol Cell Biol 1990 ; 10 : 3569-77.

19. Perbal B. Caractérisation et expression du protooncogène nov humain dans les tumeurs de Wilms. Bull Cancer (Paris) 1994 ; 81: 957-61.

20. Thomopoulos G, Kyurkchiev S, Perbal B Immunocytochemical localization of NOVH and ultrastructural characteristics of $\mathrm{NCl}$ H295R cells. J Submicrosc Cytol Pathol 2001 ; 33 : 251-60.

21. Perbal B. Nuclear localization of NOV protein: a potential role for nov in the regulation of gene expression. Mol Pahol $1999 ; 52: 84-91$.

22. Hwa V, Oh Y, Rosenfeld R. The insulin-like growth factor-binding protein (IGFBP) superfamily. Endocrinol Rev 1999 ; 20 : 761-87.

23. Chou Py, Fasman GD. Prediction of protein conformation. Biochemistry $1974 ; 13: 222-45$.

24. Burren C, Wilson E, Hwa V, Oh Y, Rosenfeld R. Binding properties and distribution of insulin-like growth factor binding proteinrelated protein 3 (IGFBP$r P 3 / N o v H)$, an additional member of the IGFBP superfamily. J Clin Endocrinol Metab 1999 ; 84 : 1096-103.

25. Kim HS, Nagalla SR, Oh Y, Wilson $\varepsilon$, Roberts CT Jr, Rosenfeld RG. Identification of a family of low-affinity insulin-like growth factor binding proteins (IGFBPs): characterisation of connective tissue growth factor as a member of the IGFBP super family. Proc Natl Acad Sci USA 1997 ; 94 : 12981-6. 
26. Chevalier G, Yeger H, Martinerie $C$, et al. NovH: differential expression in developing kidney and Wilms' tumors. Am J Pathol 1998 ; 152 : 1563-75.

27. Perbal $B$, Martinerie $C$, Sainson R, Werner M, He B, Roizman $B$. The C-terminal domain of the regulatory proteinNOVH is sufficient to promote interaction with fibulin IC: a clue for a role of NOVH in celladhesion signaling. Proc Natl Acad Sci USA $1999 ; 96: 869-74$.

28. Ayer-Lelievre C, Brigstock D, Lau L, Pennica D, Perbal $B$, Yeger H. Report on the first international workshop on the CCN family of genes. Mol Pathol 2001 ; 54 : 105-7.

29. O'Brien TP, Lau LF. Expression of the growth factor-inducible immediate early gene cyr6l correlates with chondrogenesis during mouse embryonic development. Cell Growth Differ 1992 ; 3 : 645-54.

30. Babic A, Kireeva M, Kolesnikova T, Lau L. CYR61, a product of a growth factor-inducible immediate early gene, promotes angiogenesis and tumor growth. Proc Natl Acad Sci USA 1998 ; 95 : 6355-60.

31. Nakanishi T, Kimura $Y$, Tamura T, et al. Cloning of a mRNA preferentially expressed in chondrocytes by differential display-PCR from a human chondrocytic cell line that is identical with connective tissue growth factor (CTGF) mRNA. Biochem Biophys Res Commun 1997 ; 234 : 206-10.

32. Wong M, Kireeva M, Kolesnikova T, Lau L. Cyr61, Product of a growth factorinducible immediate-early gene, regulates chondrogenesis in mouse limb bud mesenchymal cells. Dev Biol 1997 ; 192 : 492-508.

33. Kocialkowski S, Yeger $\mathrm{H}$, Kingdom J, Perbal B, Schofield P. Expression of the human nov gene in first trimester fetal tissues. Anat Embryol 2001 ; 203 : 417-27.

34. Katsube KI, Chuai ML, Liu $y C$, et al. The expression of chicken nov, a member of the CCN gene family in early stage development. Gene expression pattern. Brain Res 2002; 1: 61-5.

35. Blom IE, van Dijk AJ, Wieten $L$, et al. In vitro evidence for differential involvement of CTGF, TGFbeta, and PDGF-BB in mesangial response to injury. Nephrol Dial Transplant $2001 ; 16$ : 1139-48.

36. Tsai MS, Hornby AE, Lakins J, Lupu R. Expression and function of CYR61, an angiogenic factor, in breast cancer cell lines and tumor biopsies. Cancer Res 2000 ; $60: 5603-7$.

37. Glukhova L, Angevin $\varepsilon$, Lavialle C, et al. Specific genomic alterations associated with poor prognosis in high grade renal cell carcinomas. Cancer Genet Cytogenet $2001 ; 130: 105-10$.

38. Maillard M, Cadot B, Ball Ry, et al. Differential expression of novH protooncogene in human prostate cell lines and tissues. Mol Pathol 2001 ; $54: 275-80$.

39. Frazier K, Grotendorst G. Expression of connective tissue growth factor mRNA in the fibrous stroma of mammary tumors. Int J Biochem Cell Biol 1997 ; 29 : 153-61.

40. Kireeva ML, Latinkic BV, Kolesnikova TV, et al. Cyr6l and Fispl2 are both ECM-associated signaling molecules: activities, metabolism, and localization during development. Exp Cell Res $1997 ; 233: 63-77$.

41. Scholz G, Martinerie C, Perbal B, Hanafusa $\mathrm{H}$. Transcriptional down regulation of the nov proto-oncogene in fibroblasts transformed by p60v-src. Mol Cell Biol 1996; $16: 481-6$.
42. Liu C, Liu XJ, Crowe PD, et al. Nephroblastoma overexpressed gene (NOV) codes for a growth factor that induces protein tyrosine phosphorylation. Gene 1999 ; 238 : 471-8.

43. Kireeva ML, MO FE, Yang GP Lau LF. Cyr6l, a product of a growth factor-inducible immediate-early gene, promotes cell proliferation, migration, and adhesion. Mol Cell Biol 1996 ; 16 : 1326-34.

44. Grzeszkiewicz TM, Kirschling DJ, Chen N, Lau LF. CyR6 1 Stimulates human skin fibroblast migration through integrin alpha vbeta 5 and enhances mitogenesis through integrin alpha vbeta 3 , independent of its carboxyl-terminal domain. J Biol Chem 2001 ; 276 : 1943-50.

45. Chen N, Chen CC, Lau LF. Adhesion of human skin fibroblasts to Cyr6l is mediated through integrin alpha 6 beta 1 and cell surface heparan sulfate proteoglycans. J Biol Chem 2000 ; 275 : 24953-61.

46. Chen CC, Chen N, Lau LF. The angiogenic factors Cyr6l and connective tissue growth factor induce adhesive signaling in primary human skin fibroblasts. J Biol Chem 2001 ; 276 : 10443-52.

47. Manara MC, Perbal B, Benini $S$, et al. The expression of ccn3(nov) gene in musculoskeletal tumors. Am J Pathol 2002 ; $160: 849-59$.

48. Gupta N, Wang, H, McLeod TL, et al. Inhibition of glioma cell growth and tumorigenic potential by CCN3(NOV). Mol Pathol 2001 ; 54 : 293-9.

49. Koliopanos A, Friess H, Di Mola FF, et al. Connective tissue growth factor gene expression alters tumor progression in esophageal cancer. World J Surg 2002 ; 26: 420-7.

50. Shimo T, Nakanishi T, Nishida T, et al. Involvement of CTGF, a hypertrophic chondrocytespecific gene product in tumor angiogenesis. Oncology 2001 ; 61 : 315-22.

51. Saxena N, Banerjee $S$, Sengupta K, Zoubine MN, Banerjee SK. Differential expression of WISP-1 and WISP-2 genes in normal and transformed human breast cell lines. Mol Cell Biochem 2001 ; 228 : 99-104.

52. Tsai MS, Bogart DF, Li P, Mehmi L, Lupu R. Expression and regulation of cyr6l in human breast cancer cell lines. Oncogene 2002 ; 21 : 964-73.

53. Xie D, Nakachi $K$, Wang $H$, Elashoff R, Koeffer HP. Elevated levels of connective tissue growth factor, WISPl and CYR6 in primary breast cancers associated with more advanced features. Cancer Res 2001 ; 15 : 8917-23.

54. Kleer $C$, Zhang $Y$, Pan $Q$, et al. Wisp3 is a novel tumor suppressor gene of inflammatory breast cancer. Oncogene 2002 (sous presse).

55. Leng $\varepsilon$, Malcom T, Tai G, Estable M, Sadowski I. Organization and expression of the cyr6l gene in normal human fibroblasts. J Biomed Sci $2002 ; 9: 59-67$.

56. Tanaka S, Sugimachi K, Saeki $\mathrm{H}$, et al. A novel variant of WISPl lacking a von Willebrand type $C$ module overexpressed in scirrhous gastric carcinoma. Oncogene $2001 ; 20$ : 5525-32. 